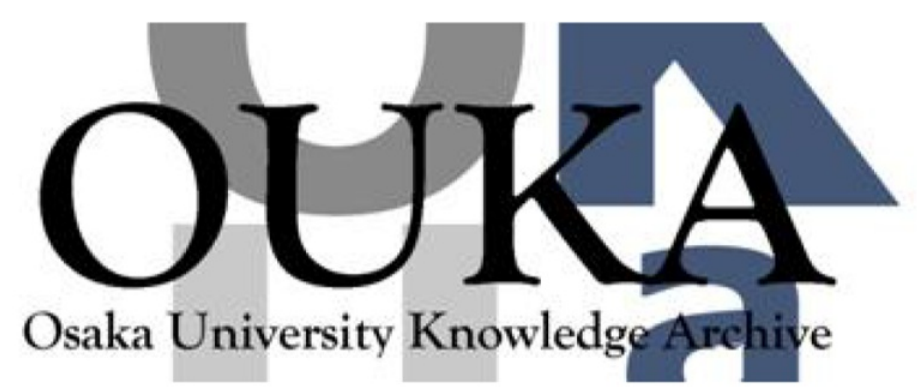

\begin{tabular}{|c|l|}
\hline Title & $\begin{array}{l}\text { Mechanism of elastic softening behavior in a } \\
\text { superlattice }\end{array}$ \\
\hline Author(s) & $\begin{array}{l}\text { Nakamura, Nobutomo; Ogi, Hirotsugu; Yasui, } \\
\text { Takeshi et al. }\end{array}$ \\
\hline Citation & $\begin{array}{l}\text { Physical Review Letters. 99(3) p.035502-1- } \\
\text { p.035502-4 }\end{array}$ \\
\hline Issue Date & $2007-07-19$ \\
\hline oaire:version & VoR \\
\hline URL & https://hdl. handle. net/11094/84183 \\
\hline rights & Copyright 2007 by the American Physical Society \\
\hline Note & \\
\hline
\end{tabular}

Osaka University Knowledge Archive : OUKA

https://ir. Library. osaka-u. ac. jp/

Osaka University 


\title{
Mechanism of Elastic Softening Behavior in a Superlattice
}

\author{
Nobutomo Nakamura, ${ }^{*}$ Hirotsugu Ogi, Takeshi Yasui, Makoto Fujii, and Masahiko Hirao \\ Graduate School of Engineering Science, Osaka University, Machikaneyama 1-3, Toyonaka, Osaka 560-8531, Japan
}

(Received 10 January 2007; published 19 July 2007)

\begin{abstract}
We measured the out-of-plane elastic constants $C_{33}$ of a $\mathrm{Co} / \mathrm{Pt}$ superlattice by picosecond ultrasound, and found that they were closely related to the thickness ratio of $\mathrm{Co}$ and Pt layers; $C_{33}$ was smaller than the prediction from the bulk values except for a specific thickness ratio. This behavior can be explained by the weak bonding at the interfaces that occurs to reduce the elastic strain energy, not by the interfacial strain. This view explained the relationship among $C_{33}$, the elastic strain energy, and perpendicular magnetic anisotropy.
\end{abstract}

PACS numbers: $62.25 .+\mathrm{g}, 43.58 .+\mathrm{z}, 75.50 . \mathrm{Ss}$

The superlattice has been attracting a lot of attention because of its anomalous properties. Among them, its property of elasticity has been investigated since the report of the supermodulus effect [1]. Although many researchers reached negative results from experiments [2-6] and atomistic-based simulations [7,8], it is still an open question. Among the independent elastic constants, $C_{i j}$, the outof-plane elastic constant $C_{33}$ showed a common behavior in several superlattice systems: as the bilayer thickness $\Lambda$ decreased, $C_{33}$ drastically decreased $[9,10]$. The previous studies concluded that $C_{33}$ was closely related with the lowered interface stiffness due to the lattice misfit. However, there are other possible mechanisms such as the alloy phase and weak-bonding regions at the interfaces which have not been sufficiently discussed, and their contributions need to be reconsidered. This Letter aims to clarify the mechanism of the anomalous elastic behavior of superlattice and establish the comprehensive elastic model that can consistently explain the elastic property, as well as the magnetic property, of superlattice.

We studied Co/Pt superlattice for three reasons. First, it involves a large lattice misfit and high binding affinity between $\mathrm{Co}$ and $\mathrm{Pt}$ to make the coherent interfaces. The interatomic distance of $\mathrm{Pt}$ is larger than that of Co by $10.7 \%$, and the lattice misfit introduces elastic strain larger than 0.05 in magnitude. This strain may be large enough to modify $C_{i j}$ through the anharmonic interatomic potential. Second, the biaxial Poisson's ratio of Pt is large [11], indicating that the Pt film is easily deformed in the outof-plane direction by the in-plane biaxial strain. The above two characteristics of $\mathrm{Co} / \mathrm{Pt}$ superlattice are suitable for evaluating the contribution of the strain dependence of the modulus. Third, Co/Pt superlattice shows high perpendicular magnetic anisotropy (PMA), and the lattice coherency is easily evaluated by the degree of PMA. The easy magnetization direction of magnetic thin film is usually parallel to the film surface because of predominance of the shape-magnetic anisotropy [12]. However, in some superlattices, it is perpendicular to the film surface when the thickness of the magnetic material is of the order of angstrom, which is called PMA [13]. The Co/Pt superlattice is the well-known system showing strong PMA, for which large elastic strain at the interfaces is indispensable $[14,15]$. Therefore, the Co/Pt superlattice is an ideal system for clarifying the mechanism of the anomalous elasticity.

A series of $\mathrm{Co} / \mathrm{Pt}$ superlattice thin films was deposited on (001) Si substrates by magnetron sputtering. A Pt-buffer layer of $16 \AA$ was first deposited. In order to change the strain in $\mathrm{Co}$ and $\mathrm{Pt}$ layers, we varied the thickness ratio between Co and Pt. The Co-layer thickness of six specimens was fixed to be $4 \AA$, and the Pt-layer thickness was changed to be $2,4,8,12,16$, and $20 \AA$. We deposited the films on several substrates simultaneously to confirm reproducibility. Two other specimens had a thickness ratio of $d_{\mathrm{Co}}: d_{\mathrm{Pt}}=1: 4$, and the bilayer thicknesses $\Lambda\left(=d_{\mathrm{Co}}+\right.$ $d_{\mathrm{Pt}}$ ) were 100 and $200 \AA$. The number of bilayers was changed to keep the total film thickness of $800 \AA$ for all eight specimens. Bilayer thickness was determined by the $\mathrm{x}$-ray total reflectivity measurement [16], and the total film thickness was determined from the bilayer thickness and the number of the bilayers. For all specimens, we observed sharp satellite peaks, which confirmed good periodicity.

The picosecond-laser ultrasound technique found by Thomsen et al. [17] enables one to determine the roundtrip time of coherent acoustic phonons in $\mathrm{GHz}$ frequencies propagating in the film-thickness direction of a thin film. Film's $C_{33}$ is determined from the round-trip time $\Delta t$, the mass density $\rho$, and the film thickness $d$, through $C_{33}=$ $\rho(2 d / \Delta t)^{2}$. We used the pulse-laser beam of $800 \mathrm{~nm}$ wavelength with 100 -fs duration. It was separated into the pump and probe beams with power of 10 and $5 \mathrm{~mW}$, respectively. Figure 1 shows the typical responses of the reflectivity variation detected by the probe beam; we observe five echoes of the longitudinal wave. After eliminating the background due to thermal phonons, we calculated the autocorrelation function with the first echo as the reference; the fifth echo is clearly visible. By this means, we yielded $\Delta t$ within the error limit of $2 \%$.

We evaluated PMA by measuring the effective magnetic anisotropy energy $K_{\text {eff }}\left(=U_{\|}-U_{\perp}\right)$, where $U_{\|}$is the 


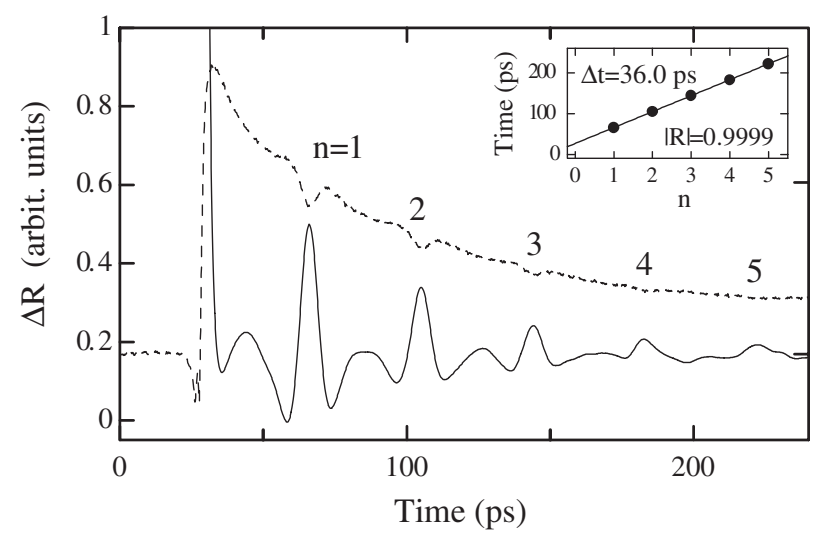

FIG. 1. Reflectivity change determined by the probe beam. As-measured multiple reflection echoes in $\operatorname{Pt}(16 \AA)$ / $[\mathrm{Co}(4 \AA) / \operatorname{Pt}(8 \AA)]_{67}$ are denoted by the broken line, and the autocorrelation function of the observed data by the solid line. $\Delta t$ is determined from the relationship between $n$ and the individual transit times (see inset).

total energy of the system per unit volume of Co with the in-plane magnetization, and $U_{\perp}$ is that with the out-ofplane magnetization. When $U_{\perp}<U_{\|}, K_{\text {eff }}$ becomes positive, and the superlattice shows PMA. $K_{\text {eff }}$ is determined from the area surrounded by the initial-magnetization curves for the in-plane and out-of-plane directions [15]. Figure 2(a) shows the relationship between $\Lambda$ and $C_{33}$ of Co/Pt superlattice. $C_{33}$ shows the maximum at $\Lambda=12 \AA$ which has never been observed in any superlattice systems. Figure 2(b) shows the relationship between $\Lambda$ and $K_{\text {eff }}$. The vertical axis is normalized by the shape-magnetic-

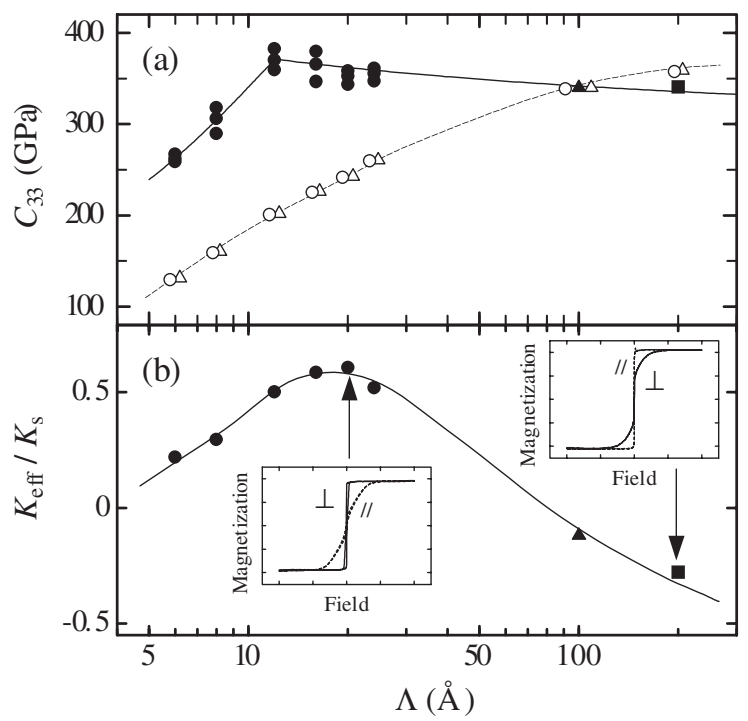

FIG. 2. Bilayer thickness dependence of $C_{33}$ and $K_{\text {eff }}$. (a) Solid marks denote the measured $C_{33}$ of $\mathrm{Co} / \mathrm{Pt}$ superlattice; circles, triangles, and squares indicate the Co-layer thickness of 4, 20, and $40 \AA$, respectively. Open circles and triangles denote the modulus calculated by the interfacial-strain-dependent model of $\mathrm{Co}(\mathrm{hcp}) / \mathrm{Pt}$ and $\mathrm{Co}(\mathrm{fcc}) / \mathrm{Pt}$ superlattice, respectively. (b) $K_{\text {eff }}$ normalized by $K_{s}$. The insets show the typical magnetic hysteresis loops. anisotropy energy $K_{s}\left(=N I_{s}^{2} / 2 \mu_{0}\right)$ [12] $\left(I_{s}\right.$ : saturation magnetization; $N$ : demagnetization factor of thin film $(N=1) ; \mu_{0}$ : permeability of vacuum). $K_{\text {eff }}$ shows large values for $12 \AA<\Lambda<24 \AA$ and PMA disappears when $\Lambda>100 \AA$. Considering that a large strain is required to achieve high PMA, Co/Pt superlattice films of $\Lambda=$ 6-24 A have grown epitaxially at the interfaces, but the other two thicker films barely grow epitaxially. Figure 3 plots the measured $C_{33}$ normalized by the bulk value calculated for $\mathrm{Co}(\mathrm{hcp}) / \mathrm{Pt}$ superlattice using the rule of mixture, $C_{33}^{\text {bulk }}=\left(f_{\mathrm{Co}} / C_{33}^{\mathrm{Co}}+f_{\mathrm{Pt}} / C_{33}^{\mathrm{Pt}}\right)^{-1}$, where $f_{i}$ is the volume fraction of the component $i$. For this, we used the reported $C_{i j}$ of bulk hcp-Co [18] and Pt [19]. At room temperature, bulk Pt takes fcc structure, and (111) plane is the close-packed plane. In bulk Co, hcp is the stable structure at room temperature. However, in $\mathrm{Co} / \mathrm{Pt}$ superlattice thin films, some reports suggest that fcc $\mathrm{Co}$ is stabilized when its thickness is less than $4 \AA[20,21]$. Close-packed planes of hcp and fcc Co are (0001) and (111) planes, respectively. In the following discussion, we therefore consider both hcp and fcc Co for Co layers.

We consider three mechanisms that can affect $C_{33}$. The first is the interfacial strain, which has been considered as the dominant factor for the softened superlattice. Clemens and Eesley [9] predicted the strain-dependent $C_{33}^{s}$ by the

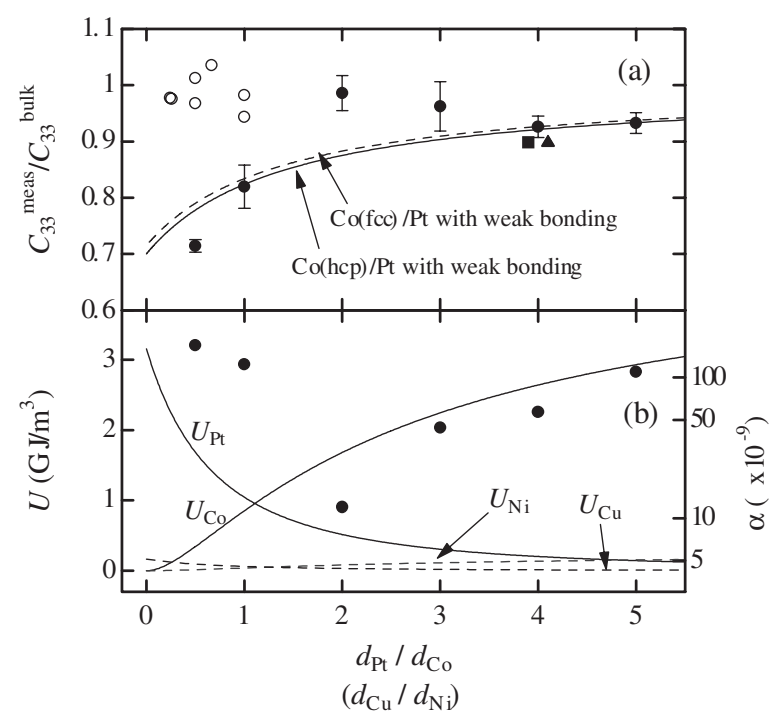

FIG. 3. Dependence of $C_{33}$ and strain energy on the thickness ratio of $\mathrm{Co} / \mathrm{Pt}$ and $\mathrm{Cu} / \mathrm{Ni}$ superlattices. (a) Vertical axis is normalized by $C_{33}$ of $\mathrm{Co}(\mathrm{hcp}) / \mathrm{Pt}$ or $\mathrm{Cu} / \mathrm{Ni}$ superlattices. Solid and open symbols describe the measurements of $\mathrm{Co} / \mathrm{Pt}$ and $\mathrm{Cu} / \mathrm{Ni}$ superlattice, respectively. Solid and dashed lines are the normalized $C_{33}$ of $\mathrm{Co}(\mathrm{hcp}) / \mathrm{Pt}$ and $\mathrm{Co}(\mathrm{fcc}) / \mathrm{Pt}$ superlattice calculated by the micromechanics theory, respectively. (b) Solid circles denote the degree of the volume fraction of the weakbonding region $\alpha$ that gives the best fit to the measured $C_{33}$ of $\mathrm{Co} / \mathrm{Pt}$ superlattice. Solid lines denote strain energy in $\mathrm{Co}$ and $\mathrm{Pt}$ layers per unit volume of $\mathrm{Co}$ and $\mathrm{Pt}$, respectively, and dashed lines are those of $\mathrm{Cu}$ and $\mathrm{Ni}$ layers per unit volume of $\mathrm{Cu}$ and $\mathrm{Ni}$, respectively. 
following equation assuming that the local $C_{33}$ at the interfaces is lowered by the expansion of the interatomic distance.

$$
\frac{C_{33}^{s}}{C_{33}^{0}}=\left\{1-\frac{2 m_{i} d_{i}}{\Lambda}\left(1-\frac{2}{C_{33}^{i}} \frac{C_{33}^{\mathrm{Co}} C_{33}^{\mathrm{Pt}}}{C_{33}^{\mathrm{Co}}+C_{33}^{\mathrm{Pt}}}\right)\right\}^{-1} .
$$

Where $C_{33}^{0}$ is the macroscopic $C_{33}$ without the interfacial softening. $C_{33}^{\mathrm{Co}}$ and $C_{33}^{\mathrm{Pt}}$ are those of bulk $\mathrm{Co}$ and $\mathrm{Pt}$, respectively. $m_{i}$ is the number of the layers where elastic softening occurs. $d_{i}$ is the interatomic distance at the interfaces. $C_{33}^{i}$ is the out-of-plane modulus at the interfaces, which is deduced by considering the adhesion between different metals as $C_{33}^{i} / C_{33}^{e}=2 / e^{a^{*}}-\left(1+a^{*}\right) / e^{a^{*}}$ [22], where $a^{*}$ is a scaled length defined by $a^{*}=\left(a-a_{m}\right) / l$. $a$, $a_{m}$, and $l$ are the actual interatomic distance along the filmthickness direction, the average interatomic distance of Co and $\mathrm{Pt}$, and a scaling length, respectively. $C_{33}^{e}$ is the equilibrium interface elastic constant. $a$ is measurable from the $\mathrm{x}$-ray diffraction spectra [9], and $l$ is given by Rose et al. [22]. According to this model, we calculated $C_{33}$, which is plotted in Fig. 2(a) by open marks. They are, however, much smaller than the measurements for $\Lambda=6-24 \AA$. Furthermore, PMA decreases when $\Lambda<10 \AA$ indicating low lattice distortion, which would have caused a larger modulus if the interfacial-strain effect were dominant. Therefore, the interfacial-strain effect cannot be the dominant mechanism for $C_{33}$ 's behavior.

Second is the intermetallic phase at the interfaces. There is a possibility of interdiffusion and formation of the CoPt intermetallic alloy at the interfaces [23]. Bandhu et al. [24] measured $C_{i j}$ of $\mathrm{Co}_{3} \mathrm{Pt}$ thin films, and they observed smaller $C_{33}$ than that predicted from bulk $C_{33}$ of Co and Pt: $C_{33}^{\mathrm{Co}_{3} \mathrm{Pt}}=308 \mathrm{GPa}, C_{33}^{\mathrm{Pt}(111)}=385 \mathrm{GPa}, C_{33}^{\mathrm{fcc}-\mathrm{Co}(111)}=$ $367 \mathrm{GPa}$, and $C_{33}^{\mathrm{hcp}-\mathrm{Co}}=357 \mathrm{GPa}$. This result implies that the existence of $\mathrm{Co}_{3} \mathrm{Pt}$ interatomic alloy decreases the macroscopic $C_{33}$ of $\mathrm{Co} / \mathrm{Pt}$ superlattice. However, the rule of mixture predicted the decrease of the $C_{33}$ by $15 \%$ for $\Lambda=6$ and $8 \AA$ at most even if all the Co layers were replaced into the alloy phase by the interdiffusion. Therefore, the $\mathrm{Co}_{3} \mathrm{Pt}$ alloy cannot quantitatively explain the decreases of $C_{33}$ at $\Lambda=6$ and $8 \AA$. (Observed moduli were decreased by $20-30 \%$.) Furthermore, considering that the alloy phase arises at the interfaces and its volume fraction is proportional to the number of the interfaces, alloy phase cannot explain the peak of $C_{33}$ at $\Lambda=12 \AA$.

The third possibility is the formation of weak-bonding regions at the interfaces to relax the huge lattice-misfit strain. They decrease the macroscopic $C_{i j}$. We estimate this effect using the micromechanics theory considering that the $\mathrm{Co} / \mathrm{Pt}$ superlattice is a multiphase composite consisting of Pt matrix and inclusions of $\mathrm{Co}$ and weak-bonding regions. We assume the $\mathrm{Pt}$ matrix to be isotropic for simplicity because an anisotropic matrix requires numerical calculations with many parameters for the Eshelby tensor [25]. This assumption is, however, acceptable, con- sidering that we are interested in the relative change of the composite $C_{33}$ for the $\Lambda$. Also, the elastic anisotropy factor of $\mathrm{Pt}$ is 1.6 and close to unity for an isotropic material, which supports this approximation. Co layers are assumed to be penny-shape inclusions, and the weak-bonding regions are replaced by the penny-shape microcracks. (Here, we neglect the effect of interfacial dislocations and delaminations on $C_{i j}$, because their effect is usually smaller than that of the microcrack while they release interfacial stress, and they are rather the origin of formation of the microcrack.) Minor axes of both inclusions are perpendicular to the film surface. We calculated the isotropic $C_{i j}$ of $\mathrm{Pt}$ matrix using the Hill averaging method [26]. The rotation-symmetry axis of $\mathrm{Co}$ is set to be parallel to the minor axis of the penny-shape inclusions. The elastic constant matrix of the composite $\mathbf{C}_{\mathrm{Comp}}$ is then expressed by

$$
\begin{aligned}
\mathbf{C}_{\mathbf{C o m p}}= & {\left[f_{m} \mathbf{C}_{\mathbf{m}}+f_{I_{1}} \mathbf{C}_{\mathbf{I}_{1}} \mathbf{A}_{\mathbf{I}_{1}}+f_{I_{2}} \mathbf{C}_{\mathbf{I}_{2}} \mathbf{A}_{\mathbf{I}_{2}}\right] } \\
& \times\left[f_{m} \mathbf{I}+f_{\mathbf{I}_{1}} \mathbf{A}_{\mathbf{I}_{1}}+f_{\mathbf{I}_{2}} \mathbf{A}_{\mathbf{I}_{2}}\right]^{-1} .
\end{aligned}
$$

Here, $\mathbf{I}$ is the unit matrix. Subscripts, $m, I_{1}$, and $I_{2}$ denote the matrix and inclusions, respectively. $\mathbf{C}_{\mathbf{i}}$ and $\mathbf{A}_{\mathbf{i}}$ are the elastic constant matrix and strain concentration factor of constitute $i$, respectively. According to the equivalentinclusion theory [27] and the mean-field theory [28], $\mathbf{A}_{\mathbf{i}}$ can be written as $\mathbf{A}_{\mathbf{i}}=\left[\mathbf{S}_{\mathbf{i}} \mathbf{C}_{\mathbf{m}}^{-1}\left(\mathbf{C}_{\mathbf{i}}-\mathbf{C}_{\mathbf{m}}\right)+\mathbf{I}\right]^{-1}$ [25]. The Eshelby tensor $\mathbf{S}$ is given in terms of Poisson's ratio of the matrix and the aspect ratio of the inclusions.

The key parameters are the aspect ratios and volume fractions of the inclusions, which are closely related to each other. In order to approximate Co layers by pennyshape inclusions, the aspect ratio must be significantly small. Here we assume the aspect ratio to be $1.0 \times 10^{-8}$ from the in-plane length of the specimen and Co-layer thickness. The aspect ratio of the microcrack is also assumed to be $1.0 \times 10^{-8}$. The volume fraction of $\mathrm{Co}$ is determined from the thickness ratio between $\mathrm{Co}$ and $\mathrm{Pt}$ layers. Although the volume fraction of the microcracks is uncertain, it should be proportional to the number of interfaces. Therefore, we assume it to be $\alpha \times f_{\mathrm{Co}}$. $\alpha$ is the proportionality constant and it is the only fitting parameter.

First, we assumed $\alpha$ to be independent of the thickness ratio, determined it by fitting the calculation to the measurement at $d_{\mathrm{Pt}} / d_{\mathrm{Co}}=5\left(\alpha\right.$ was $\left.1.1 \times 10^{-7}\right)$, and compared the calculated $C_{33}$ with the measurement in Fig. 3(a). As $d_{\mathrm{Pt}} / d_{\mathrm{Co}}$ decreases, $C_{33}$ becomes smaller, because the volume fraction of the interface increases and the weakbonding region increases. Calculated $C_{33}$ shows good agreement with measured $C_{33}$ at $d_{\mathrm{Pt}} / d_{\mathrm{Co}}=0.5,1,4$, and 5 , and we found that only the weak-bonding regions can explain the softening of the film at the small thickness ratio. However, we observe the discrepancies for $d_{\mathrm{Pt}} / d_{\mathrm{Co}}=2$ and 3 .

We investigate the origin of the difference at $d_{\mathrm{Pt}} / d_{\mathrm{Co}}=$ 2 and 3 by considering the relationship between the elastic 
strain energy and $\alpha$. The volume fraction of the weakbonding regions must change depending on the thickness ratio. Therefore, we deduced $\alpha$ that gives the best fitting with the measured $C_{33}$, which are shown in Fig. 3(b) by solid circles. Then, assuming the complete bonding at the interfaces, we calculated the elastic strains of individual layers with the conventional continuum theory for the biaxial in-plane stress case. The strain energy per unit volume, $U$, is given by $U=\sigma \epsilon$, where $\sigma$ and $\epsilon$ are the biaxial in-plane stress and strain, respectively. Figure 3(b) shows the result. As $d_{\mathrm{Pt}} / d_{\mathrm{Co}}$ increases, $U_{\mathrm{Co}}$ increases and $U_{\mathrm{Pt}}$ decreases, and they intersect between $d_{\mathrm{Pt}} / d_{\mathrm{Co}}=1$ and 2. Being close to this point, $\alpha$ takes a minimum. This result suggests that when $d_{\mathrm{Pt}} / d_{\mathrm{Co}}$ is smaller, weak-bonding regions are induced to decrease $U_{\mathrm{Pt}}$, and when it is larger, they are induced to decrease $U_{\mathrm{Co}}$. At the same time, this result denies the conventional interpretation that the interfacial strain is the dominant factor for the lowered modulus in the superlattice systems. If it were the case, $C_{33}$ should have shown a minimum around $d_{\mathrm{Pt}} / d_{\mathrm{Co}} \sim 2$, because when the volume fraction of the weak-bonding regions is the smallest, the strain at the interfaces becomes the largest. From these discussions, we attribute the lowered $C_{33}$ to the weak-bonding regions between the $\mathrm{Co}$ and $\mathrm{Pt}$ layers. Considering that large strain caused by the lattice misfit is indispensable for PMA [14,15], negative $K_{\text {eff }}$ indicates that when $\Lambda$ is larger than $100 \AA, \mathrm{Co} / \mathrm{Pt}$ superlattice barely grows epitaxially, and $C_{33}$ can decrease at the interfaces. However, $C_{33} / C_{33}^{\text {bulk }}$ at $\Lambda=100$ and $200 \AA$ is close to that at $\Lambda=20 \AA$ because of the smaller volume fraction of the interface.

In order to confirm the validity of the above discussion, we further measured $C_{33}$ of the $\mathrm{Cu} / \mathrm{Ni}$ superlattice system. They were deposited on monocrystal Si substrates at the same condition as $\mathrm{Co} / \mathrm{Pt}$ superlattice, and the thickness ratio of $\mathrm{Cu}$ to $\mathrm{Ni} d_{\mathrm{Cu}} / d_{\mathrm{Ni}}$ was ranged from 0.25 to 1 $\left[d_{\mathrm{Cu}}(\AA) / d_{\mathrm{Ni}}(\AA)=50 / 50,10 / 10,20 / 80,4 / 16,10 / 20\right.$, $5 / 10$, and $10 / 15]$. The number of bilayers was changed to keep the total film thickness of $800 \AA$ for all specimens. $C_{33}$ of $\mathrm{Cu} / \mathrm{Ni}$ superlattice is plotted in Fig. 3, which are normalized by $C_{33}$ predicted from the bulk materials' $C_{33}$ $[29,30]$. Comparing to $\mathrm{Co} / \mathrm{Pt}$ superlattice, significant softening was not observed even at the very small thickness ratio. Considering the elastic strain energy in $\mathrm{Cu}$ and $\mathrm{Ni}$ layers, this result is reasonable. In the $\mathrm{Cu} / \mathrm{Ni}$ system, lattice mismatch at the interfaces is $2.6 \%$, being smaller than that of the $\mathrm{Co} / \mathrm{Pt}$ system, and $C_{33}$ of $\mathrm{Cu}$ and $\mathrm{Ni}$ is smaller than those of $\mathrm{Co}$ and $\mathrm{Pt}$. Therefore, elastic strain energy in $\mathrm{Cu} / \mathrm{Ni}$ superlattice becomes smaller than that in $\mathrm{Co} / \mathrm{Pt}$ superlattice [see the broken lines in Fig. 3(b)]. Thereby, the weak-bonding regions are barely induced at the interfaces, and the macroscopic $C_{33}$ is comparable to the predictions.

Two important findings were observed in this study. One is the above-discussed strain-energy dependence of $C_{33}$ of superlattice, and the other is the relationship between the elastic constants and PMA. Despite many studies, the origin of PMA has not been clarified yet, because of a number of possible factors and the ambiguity of bonding condition at the interfaces. However, the measurement of $C_{33}$ enables one to predict the bonding condition through softening of $C_{33}$, which is a new approach for discussing the relationship between PMA and the interfacial bonding. Furthermore, it enables one to evaluate PMA nondestructively. Considering that picosecond-laser ultrasounds is a noncontacting technique, PMA can be evaluated during the deposition process in principle, and is applicable for industrial fields.

We are grateful to Dr. S. Morimoto, Osaka University, and Low Temperature Center, Osaka University, for the measurement of the magnetic properties.

*nobutomo@me.es.osaka-u.ac.jp

[1] W. M. C. Yang et al., J. Appl. Phys. 48, 876 (1977).

[2] M. R. Khan et al., Phys. Rev. B 27, 7186 (1983).

[3] B. M. Davis et al., Phys. Rev. B 43, 9304 (1991).

[4] K. Sakaue et al., J. Magn. Magn. Mater. 126, 207 (1993).

[5] H. Huang and F. Spaepen, Acta Mater. 48, 3261 (2000).

[6] P. Villain et al., Appl. Phys. Lett. 81, 4365 (2002).

[7] I. K. Schuller and A. Rahman, Phys. Rev. Lett. 50, 1377 (1983).

[8] D. Wolf and J.F. Lutsko, J. Appl. Phys. 66, 1961 (1989).

[9] B. M. Clemens and G. L. Eesley, Phys. Rev. Lett. 61, 2356 (1988).

[10] E. E. Fullerton et al., J. Appl. Phys. 73, 7370 (1993).

[11] H. Ogi et al., Phys. Rev. Lett. 98, 195503 (2007).

[12] S. Chikazumi, Physics of Magnetizm (Wiley, New York, 1964).

[13] P. F. Carcia et al., Appl. Phys. Lett. 47, 178 (1985).

[14] T. Kingetsu et al., Sci. Tech. Adv. Mater. 2, 331 (2001).

[15] Y. Kamada et al., J. Appl. Phys. 90, 5104 (2001).

[16] H. Kiessig, Ann. Phys. (Leipzig) 10, 769 (1931).

[17] C. Thomsen et al., Phys. Rev. B 34, 4129 (1986).

[18] E. S. Fisher and D. Dever, Trans. Metall. Soc. AIME 239, 48 (1967).

[19] R. E. Macfarlane et al., Phys. Lett. 18, 91 (1965).

[20] Z. G. Lin et al., J. Appl. Phys. 73, 2433 (1993).

[21] E. Lundgren et al., Phys. Rev. B 62, 2843 (2000).

[22] J. H. Rose et al., Phys. Rev. B 28, 1835 (1983).

[23] P. C. Mclntyre et al., J. Appl. Phys. 81, 637 (1997).

[24] R. S. Bandhu et al., J. Appl. Phys. 91, 2737 (2002).

[25] T. Mura, Micromechanics of Defects in Solids (Martinus Nijhoff, Dordrecht, 1987), 2nd ed.

[26] R. Hill, Proc. Phys. Soc. London Sect. A 65, 349 (1952).

[27] J. D. Eshelby, Proc. R. Soc. A 241, 376 (1957).

[28] T. Mori and K. Tanaka, Acta Metall. 21, 571 (1973).

[29] H. Ogi et al., J. Acoust. Soc. Am. 106, 660 (1999).

[30] H. Ledbetter and S. Kim, in Handbook of Elastic Properties of Solids, Liquids, and Gases (Academic, New York, 2001), Vol. 2. 